\title{
Regeneration des Kropfes.
}

\author{
Von \\ Teruwo Kuriyama. \\ Aus dem Anatomischen Institut der Kaiserlichen Universität zu Kioto.
}

Mit 7 Figuren auf Tafel XXXIX.

Der Oesophagus von bestimmten Vögeln zeigt in der Gegend des Brusteinganges eine Art Ausbuchtung, welche als Kropf bezeichnet wird. Die Kropfwand besitzt Schleimdrüsen wie die anderen Oesophagusteile. Dieser Kropf spielt als Mazerierbehälter für trockene und schwer verdauliche Nahrung eine wichtige Rolle. Es findet sich in der Literatur noch keine Erklärung dafür, warum der Kropf bei bestimmten Vögeln existiert und bei anderen fehlt, obgleich das Futter bei beiden Arten von Vögeln mehr oder weniger dasselbe ist. Während die Untersuchungen am Kropf bisher physiologisch oder anatomisch-histologisch angestellt worden sind, liegt, soviel ich weiss, noch keine Mitteilung darüber vor, zu welchen morphologischen Veränderungen es durch die totale oder partielle Exstirpation des Kropfes an der betreffenden Stelle kommt. Daher habe ich mich mit diesbezüglichen Experimenten an Hühneru und Tauben beschäftigt.

\section{Material und Methode.}

Als Material benützte ich Hühner und Tauben. Die Grösse des Kropfes ist sowohl nach dem Füllungszustande als auch individuell ziemlich verschieden. Bei den erwachsenen Hühnern zeigt der Kropf einen einfach kugligen Sack, der bei den jungen Hühnchen schwächer ausgebildet ist. Bei den Tauben bildet er einen beiderseits symmetrisch ausgebuchteten Sack, welcher durch eine seichte Furche in der Medianlinie unvollständig geteilt ist.

Was die Hühner ambetrifft, so habe ich sowohl Hühnchen am ersten Tage nach der Ausbrütung als auch erwachsene, über 100 Tage alte 
gebraucht. Bei den Tauben verwendete ich ausschliesslich erwachsene, bei denen ich den Kropf total und halbseitig (links) exstirpierte.

Die Anzahl der nach der Operation am Leben gebliebenen Exemplare war folgende :

Bei totaler Exstirpation des Kropfes bei erwachsenen Hühnern...... 4 Fälle,

bei totaler Exstirpation des Kropfes bei jungen Hühnchen.....12 Fälle,

bei totaler Exstirpation des Kropfes bei erwachsenen Tauben...... 5 Fälle,

bei halbseitiger Exstirpation des Kropfes bei erwachsenen Tauben...... 4 Fälle,

Gesamtsumme der Fälle 25.

Die Operation wurde unter Chloroformnarkose aseptisch ausgeführt. Ich legte zunächst an der Haut in der Kropfgegend einen Längsschnitt an und exstirpierte dam den Kropf von seinem Wurzelteile aus, wodurch der operierte Oesophagusteil nach dem Anlegen der Naht genaue Rohrform aufwies (Fig. 6.). Besonders achtete ich darauf, dass die Seitenwand des Kropfes nicht zurückblieb. Bei der halbseitigen Resektion des Taubenkropfes legte ich durch die ganze Länge des Organs einen Medianschnitt, exstirpierte den linksseitigen Kropfteil und liess den rechtsseitigen zurück.

Die Fixation geschah in Formolwasser. Beobachtet wurde hauptsächlich makroskopisch, doch auch zeitweise mikroskopisch mittels der Haematoxylineosin-Doppelfärbung.

\section{Befunde.}

Am Tage der Operation wurde dem Tiere kein Futter gegeben, sondern nur Wasser. Vom nächsten Tage an gab ich anfangs weiches, dann allmählich festeres Futter. Junge Hühnchen zeigten schon am Tage nach der Operation Fresslust. Erwachsene Hübner und Tauben liessen aber manchmal den Appetit vermissen und gingen nach einigen Tagen zugrunde, während die Tauben mit halbreseziertem Kropfe ruhig weiter gediehen. Die Untersuchung 3 Tage nach der Operation liess bei den jungen Hühnchen an der resezierten Stelle keine Neubildung der Ausbuchtumg erkemnen. Die Wunde war einfach geheilt. 10 Tage nach der Operation war eine dem normalen Kropf ähnliche Ausbuchtung zu konstatieren (Fig. 1), welche durch Ausdehnung der vorderen und lateralen Wand des operierten Oesophagusteils entstanden war, als ganzes 
eine längliche Gestalt besass und mit dem eigentlichen Oesophagus durch eine lange Basis in Verbindung stand. Dieses Ausbuchtung nahm mit den Tagen nach und nach an Grösse zu. Doch hatte sie 30 Tage nach der Operation ihre normale Grösse noch nicht erreicht, und nur ihre Form war fast normal (Fig. 2 und 3). Auch bei erwachsenen Hühnern und Tauben bildete sich 4 bis 5 Tage nach der totalen Exstirpation ani der Exstirpationsstelle eine Ausbuchtung aus. Die gebildeten Ausbuchtungen waren anfangs stets kleiner als normal, aber 30 Tage nach der Operation zeigten sie mehr oder weniger dieselbe Grösse und Form wie die normalen (Fig. 4 und 5). Wegen der Verwachsung der Kropfwand mit der Haut konnte ich bei den Tauben nicht feststellen, ob die normalerweise vorkommende mediane Furche des Kropfes auch bei der neugebildeten Ausbuchtung bemerkbar ist. Doch fällt die dem Taubenkropf eigentümliche beiderseitige starke Ausbuchtung auch bei dem neugebildeten Kropf in die Augen (Fig. 5). 40 Tage nach der Operation gediehen die Tauben ohne Besonderheiten wie die normalen.

Bei der halbseitigen Resektion des Taubenkropfes hatte sich, wie die 30 Tage nach der Operation vorgenommene Untersuchung zeigte, an der operierten Stelle kein Kropf gebildet. Auch hatte sich die zurückgebliebene Kropfhälfte nicht besonders vergrössert. Dieser Befund zeigt, dass der halbseitige Kropf der Aufgabe des ganzen Kropfes gewachsen ist.

Das Wesen der gebildeten Ausbuchtung. Normalerweise bildet der zirkuläre Muskel des Oesophagus unterhalb des Kropfes eine Art von Sphinkter, dessen Verschluss den Durchgang des Futters verhindert und die Anhäufung desselben im Kropf zu Folge hat, wo Speichel, Schleim und Fermente auf das Futter einwirken. Erst darauf geht der Inhalt nach Erschlaffung des Schliessmuskels in den Magen über. Nach Entfernung des Kropfes ist dieser Sphinkter noch immer tätig. Dadurch sammelt sich das Futter oberhalb des Spinkters an und kommt die Ausbuchtung des betreffenden Oesophagusteils zustande.

Aber man kann nicht ohne weiteres behaupten, dass die Ausbuchtung nur durch diese passive Erweiterung der Oesophaguswand zustandekommt. Bei den Menschen und Säugetieren kommt es zur pathologischen Oesophaguserweiterung entweder diffus in weiterer Ausdehnung oder durch zirkumskripte Divertikelbildung. Handelt es sich daher bei der Oesophaguserweiterung nach der Operation als einzige Ursache um Anhäufung der Futtermasse, so ist es wohl möglich, dass sich statt der zirkumskripten Ausbuchtung diffuse Erweiterung des Oesophagus ein- 
stellt. Tatsächlich aber bemerkte ich stets an der vorderen Seite des Oesophagus nur eine zircumskripte Ausbuchtung, welche sogar eine ähnliche Form wie der Kropf besass. Danach glaube ich, dass in diesem Oesophagusteil nicht nur die passive Ursache der Ausbuchtung zu suchen ist, sondern er auch aktiv die kropfartige Ausbuchtung ermöglicht.

Anderseits war die Bildung des Kropfes bei den eben ausgebrüteten Hühnchen nicht ausgeprägt, solange sie keine Nahrung aufnahmen. Erst nach der Nahrungsaufnahme kam es zu deutlicher Ausbildung des Kropfes. Danach wirkt auch die Anhäufung der Nahrungsmasse als eine Ursache der normalen Kropfbildung. Mag nun der Bildungsmechanismus der Ausbuchtung ein aktiver oder passiver sein, kann man, insofern die normale Kropfbildung sowohl aktiv als auch passiv vor sich geht und die gebildete Ausbuchtung sich gleich wie der Kropf gestaltet und funktioniert, mit gutem Grunde die neugebildete Ausbuchtung als einen regenerierten Kropf betrachten. Im Falle der halbseitigen Exstirpation des Taubenkropfes habe ich weder Neubildung der resezierten Hälfte noch V'ergrösserung der anderseitigen Hälfte bemerkt. Dies hängt vielleicht damit zusammen, dass die zurückgebliebene Hälfte allein als Kropf genügend funktioniert.

Ich untersuchte dann die oben geschilderte Ausbuchtung histologisch und fand folgendes:

Bei Hühnchen war kurze Zeit nach der Operation hindurch die Ausbuchtung nicht deutlich, sondern mehr geschrumpft und die Wand in allen Schichten verdickt. An den Exemplaren 10 Tage und länger nach der Operation zeigte sich allmähliches Deutlicherwerden der Ausbuchtung, aber Dünmerwerden der Wandung. An der vorderen Wand war die Verdickung deutlich, besonders hatte das Epithel zahlreiche koniche Zapfen in die Tiefe gesandt, welche hier und da aus hellen, zylindrischen Zellen bestanden und sogar stellenweise schon mit Lumen versehen waren und wie Drüsen aussahen. Auch die Schleimhaut und die Muskelschicht waren verdickt (Fig. 7). Die Zeichen von Entzündung waren nur gering.

Da die hier geschilderten Veränderungen an der Nahtgegend der Vorderwand besonders deutlich zu bemerken waren, könnte man sie auch für Wundheilungsvorgänge halten. Da sich aber auch bei den normalen ausgebrüteten Hühnchen die Verdickung der vorderen Kropfwand und das proliferative Eindringen des Epithels in die Tiefe, wemn auch in geringerem Grade, vorfanden, kann man die obenerwähnten des Epithels bei den operierten Tieren nicht ohne weiteres als Granulationswucherung nach der Operation ansehen. 
Im Gegenteile bin ich vielmehr davon überzeugt, dass es sich bei dem Eindringen der konischen Epithelzapfen, der drüsenartigen Umwandlung derselben und der Verdickung der Wandung um Äusserungen einer regenerativen Wucherung handelt. Da die vordere Wandung des neugebildeten Kropfes ursprünglich aus der lateralen Wandung des Oesophagus stammt, darf man nach dem histologischen Befunde Regerationsvorgänge an diesen operierten Teilen amnehmen, auch wenn Veränderungen infolge der Operation nicht ganz auszuschliessen sind.

Bei erwachsenen Hühnen und Tauben sind die entzündlichen Prozesse in der neugebildeten Kropfwand Folge der Operation. Es findet sich schon in Handbuch der normalen und pathologischen Physiologie von Marek, Bd. III, dass es bei den Kröpfen durch Eindringen von Fremdkörpern, faulenden sowie leicht gärenden Stoffen zu entzündlichen Prozessen kommt. Auch bei den regenerierten Kröpfen der erwachsenen Hühner und Tauben finden sich deutliche Entzündungszeichen vor. Auf der inneren Wand sieht man Ansammlungen von Detritusmasse mit darunterliegendem unregelmässig angeordnetem Epithel, und in der Wand stösst man in ihrer ganzen Dicke auf zahreiche pseudoeosinophile Leucocyten. Wegen der starken entzündlichen Prozesse konnte ich bei den erwachsenen Tieren histologisch keine Regenerationszeichen feststellen.

\section{Zusammenfassung.}

1) Wird der Kropf bei eben ausgebrüteten Hühnchen entfernt, so entsteht an der Exstirpationsstelle wieder eine Ausbuchtung. Der Verfasser hält diese Ausbuchtung auf Grund verschiedener Befunde für einen regenerierten Kropf.

2) Auch bei den erwachsenen Hühnern und Tauben findet sich nach der Totalentfernung des Kropfes an der Exstirpationsstelle eine Ausbuchtung. In diesen Fällen sind die Entzündungszeichen histologisch sehr deutlich zu konstatieren. Doch ist auch diese Ausbuchtung als regenerierter Kropf anzusehen.

3) Nach halbseitiger Exstirpation des Taubenkropfes entsteht an der Exstirpationsstelle keine Ausbuchtung und ist auch nichts von Vergrösserung der anderen Kropfhälfte zu sehen.

Zum Schlusse möchte ich Herm Prof. Dr. Ch. Ogawa für seine freundliche Unterstützung bei Ausführung meiner Arbeit meinen herzlichsten Dank aussprechen. 


\section{Literaturverzeichniss.}

1) Korschelt, Regeneration und Transplantation. 1. Bd. Regeneration. 1927.

2) Kurigama, Über den Oesophagus und den Kropf der Vögel, besonders über die Kropfgefässe. Japanisch. Kaibogaku Zasshi. Bd. 4. 1931.

3) Marek, Handbuch der normalen und pathologischen Physiologie. Bd. III. 1927.

\section{Erklärung der Figuren (Taf. XXXIX).}

o.O. ......oberer Oesophagus.

r.K.......regenerierter Kropf.

u.O.......unterer Oesophagus.

M.ı.......Muskelmagen.

n.K.......normaler Kropf.

Fig. 1. Der regenerierte Kropf eines jungen Hühnchens am 10. Tage nach der Operation.

Fig. 2. Der regenerierte Kropf eines jungen Hühnchens am 30. Tage nach der Operation.

Fig. 3. Der normale Kropf eines jungen Hühnchens am 20. Tage nach der Ausbuchtung.

Fig. 4. Der regenerierte Kropf eines erwachsenen Huhnes am 30. Tage nach den Operation.

Fig. 5. Der regenerierte Kropf einer erwachsenen Taube am 40. Tage nach der Operation.

Fig. 6. Der Oesophagus eines erwachsenen Huhnes gerade nach der Operation.

Fig. 7. Schittpräparat eines regenerierten Kropfes von einem jungen Hühnchen am 20. Tage nach der Operation. Vergrösserung. $16 \times$. 
FOLIA ANATOMICA JAPONICA, BD. IX.

TAF. XXXIX.

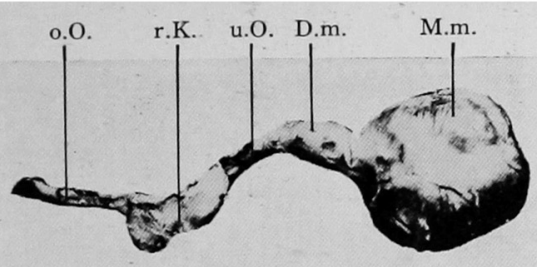

52

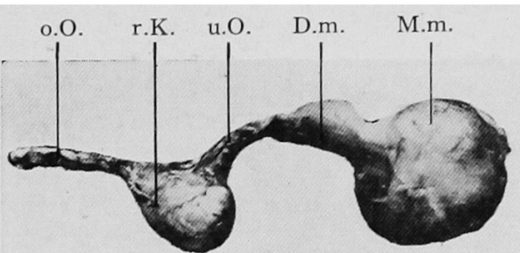

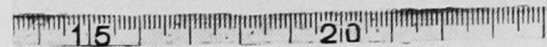

Fig. 1.

Fig. 2.

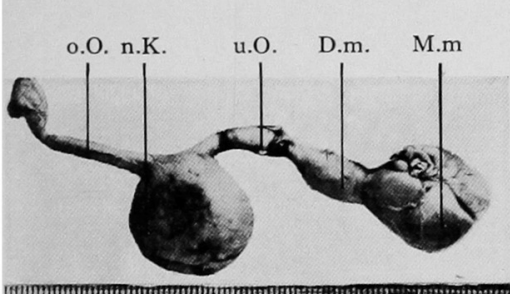

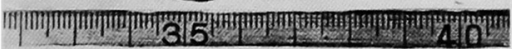

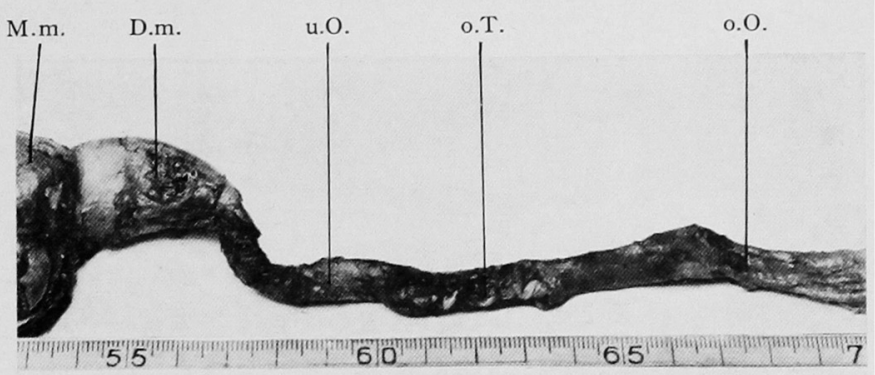

Fig. 3.
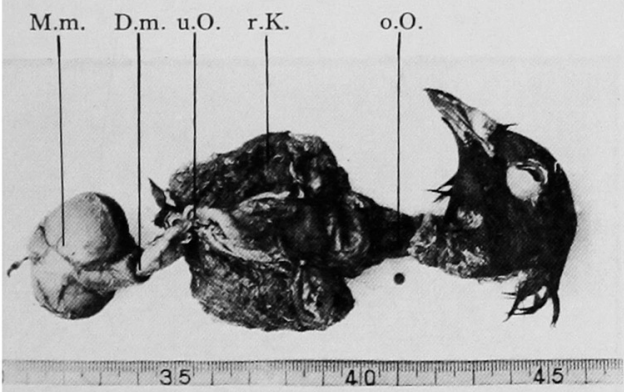

Fie. i.

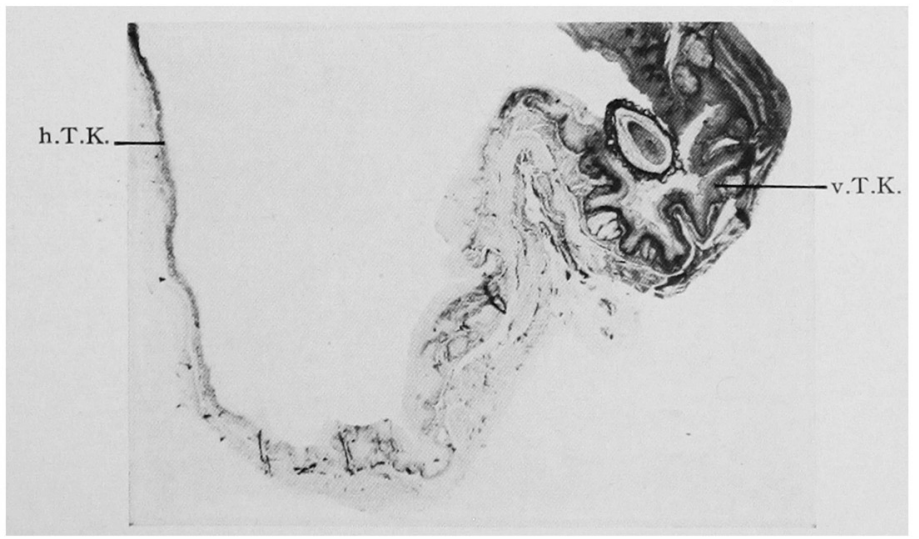

Fig. 7 .

T. Kuriyama

Fig. 4.

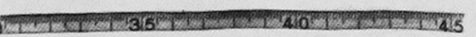

Fig. 6.

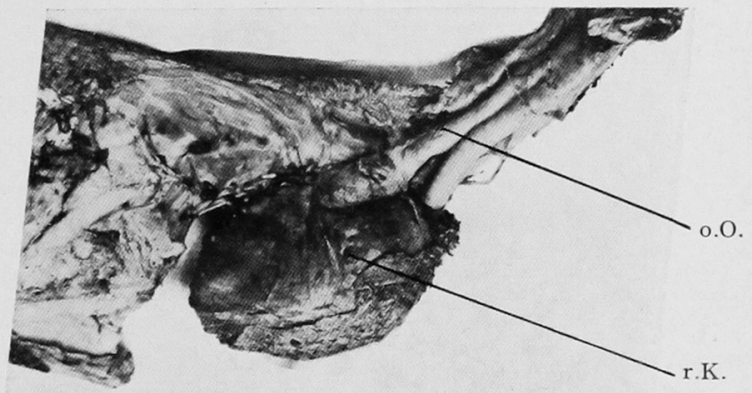

